\title{
LYDIE PARISSE
}

Université de Toulouse 2 Jean Jaurès

\section{Le théâtre intérieur de Maurice Maeterlinck dans La Mort de Tintagiles}

T a Mort de Tintagiles (1894) marque un tournant 1 dans l'œuvre de Maurice Maeterlinck. Ce drame porte la forte empreinte d'un événement déterminant dans la vie de l'auteur : la découverte des écrits du mystique flamand Ruysbroeck, dont il devint le traducteur de 1885 à 1888. Ce drame, comme tout le théâtre de Maeterlinck, permet d'interroger l'esthétique théâtrale à l'époque symboliste et dans le cadre du " théâtre sacré "1, l'une des modalités du théâtre selon Peter Brook. Selon le dramaturge anglais, le théâtre sacré questionne le sens de l'existence humaine et se veut chemin vers la vérité : "Le monde des apparences n'est qu'une écorce. Sous l'écorce, il y a la matière bouillante, pareille à celle que nous voyons à l'intérieur d'un volcan ${ }^{2}$, écrit-il dans L'Espace Vide. D'après lui, ce théâtre du verbe se marque, dans le cadre occidental et laïc, par la recherche permanente de nouvelles formes, et trouve un nouveau souffle avec des créateurs visionnaires tels Samuel Beckett. Au siècle précédent, le théâtre de Maeterlinck est marqué par une recherche à la fois sur le langage et sur l'espace dramatique qui fait de ses pièces des laboratoires du verbe mais aussi de la pensée, car ils nous confrontent à l'impensable et à l'invisible, en s'inspirant de la pensée de la voie négative ${ }^{3}$ :

\footnotetext{
${ }^{1}$ P. Brook, L'Espace vide. Écrits sur le théâtre, Paris, Seuil, 1968, p. 77.

2 Ibidem.

${ }^{3}$ Voir L. Parisse, Les Voies négatives de l'écriture dans le théâtre moderne et contemporain, Paris, Classiques Garnier, 2019. Voir aussi L. Parisse (dir.),
} 
le théâtre, en interrogeant le rapport à ce qui nous dépasse - ce qui est la caractéristique du théâtre dit " sacré »-, devient le lieu où s'expriment les voix intérieures. Interroger sur scène la présence, renouer avec un théâtre du mystère, briser les catégories enfermantes de la pensée, tels sont les quelques éléments permettant de comprendre ce théâtre, à travers la pièce La Mort de Tintagiles.

\section{Interroger sur scène la présence}

Dans les dix dernières années du XIXe siècle, Maurice Maeterlinck, à l'heure de la fondation du Théâtre Libre d'Antoine et des grandes expérimentations du théâtre naturaliste et symboliste français et européen (Ibsen, Strindberg), renouvelle considérablement le langage et l'esthétique théâtrale, inaugurant un courant qui perdure dans les esthétiques et les écritures dramatiques contemporaines. La question de la représentation devient à ses yeux une question non seulement d'ordre technique et esthétique, mais d'ordre anthropologique : elle nous renvoie à toute une phénoménologie du voir, de la vision, du visible, car au-delà de la question de la représentation, se pose celle de la présence. Représenter revient en effet toujours à évoquer ce qui est absent. En quoi le théâtre peut-il renouer avec l'univers de la présence?

Deux ans après la publication de Pelléas et Mélisande (1892), Maeterlinck écrit en 1894 Trois petits drames pour marionnettes : Intérieur ; Alladine et Palomides ; La Mort de Tintagiles. Ce dernier drame, énigmatique et réputé injouable, a été mis en scène par Vsevolod Meyerhold (auteur d'une mise en scène des plus célèbres de Pelléas en 1907) qui, convaincu de la nécessité d'un théâtre immobile, et de l'inutilité de la "vérité » à la scène, voit dans le théâtre symboliste les éléments d'une révolution radicale

Le Discours mystique dans la littérature et les arts de la fin du XIXe siècle à nos jours, Paris, Classiques Garnier, 2012. 
propres à repenser l'espace de la scène, le jeu de l'acteur et le dialogue théâtral.

Cérémonie d'une mise à mort, La Mort de Tintagiles place au premier plan le déploiement d'une force mystérieuse, puissance dévoratrice qui s'emparera de l'enfant Tintagiles, venu rejoindre ses sœurs pour les sauver de son emprise à la fois maléfique et inconnaissable : il s'agit d'une Reine réputée cruelle, accompagnée de trois servantes. L'aporie des dialogues est telle qu'aucun élément d'explication psychologique ou d'intrigue ne résiste. Sur ordre de la Reine, un enfant arrive d'une contrée inconnue et rejoint ses deux sœurs, Bellangère et Ygraine, accompagnées de l'homme au glaive, Angloval. Pendant que l'enfant dort au milieu de ses sœurs, les trois servantes de la Reine viendront le prendre et l'emmener de l'autre côté de la grande porte derrière laquelle personne encore n'a pu passer. Ce drame, comme dans tout le théâtre de Maeterlinck, cherche à substituer à la psychologie habituelle le point de vue de l'inconnaissable et à interroger le rapport de l'esthétique théâtrale à l'invisible.

La Mort de Tintagiles appartient au cycle des premières pièces, celles pour lesquelles Maeterlinck, traitant du thème récurrent de la mort, s'est vu traiter, à tort, d'auteur morbide. Peu à peu s'affirme nettement son point de vue, alimenté au regard de la spiritualité occidentale et orientale : selon lui, la mort n'existe pas en soi, ce n'est qu'un stade de métamorphoses parmi d'autres, vie et mort étant indissociables. Dans L'Oiseau bleu, publié quatorze ans plus tard, le cimetière s'évanouit devant ses visiteurs qui constatent qu' « il n'y a pas de morts ${ }^{4}$. En 1916, Maeterlinck écrit : "La vie et la mort se confondent, les vivants et les morts ne sont que des moments à peine

${ }^{4}$ M. Maeterlinck, "L'Oiseau bleu », [dans :] Idem, CEuvres III. Théâtre 2, P. Gorceix (éd.), Bruxelles, Complexe, 1999, p. 238-399. 
différents d'une existence unique et infinie et ne forment qu'une même famille immortelle $»^{5}$.

\section{Renouer avec un théâtre du mystère}

Claude Régy, qui, après Meyerhold6, a mis en scène $L a$ Mort de Tintagiles, définit ainsi la vocation du théâtre : "Réfléchir à l'incréé, à sa masse, à son immensité - dans le temps et dans l'espace - et peut-être même et surtout à sa force agissante, devrait faire partie des préoccupations majeures de ceux qui croient créer ${ }^{7}$. Dans sa mise en scène hiératique, il ira jusqu'au bout des intuitions de Meyerhold, présentant des comédiens marionnettisés seIon Edward Gordon Craig, dans un espace vide et entier, animé d'un immense store laissant filtrer de rares lueurs ${ }^{8}$. En $1906^{\circ}$, son prédécesseur russe était partagé entre deux

\footnotetext{
${ }^{5}$ M. Maeterlinck, Les Débris de la guerre, Paris, Fasquelle, 1916, p. 194.

${ }^{6}$ Vsevolod Emilievitch Meyerhold (1874-1940) a participé aux aventures théâtrales les plus radicales : symbolisme, constructivisme, révolution. II avait mis au point un vocabulaire du jeu de l'acteur : la théorie des actions physiques de l'acteur. Contre la reproduction minutieuse de la réalité pratiquée par Stanislavski, il cherchait à dénaturaliser le jeu de l'acteur, à créer un théâtre d'images, oniriques, en se concentrant sur une technique de l'extériorité. Constantin Sergueïevitch Stanislavski (1863-1938) a publié La Formation de l'acteur et La Construction du personnage. Lorsqu'il meurt en 1938, son enseignement a bouleversé toute l'Europe. Cette vision du jeu du comédien fait désormais figure de passage obligé pour tout acteur souhaitant évoluer dans le monde du théâtre ou du cinéma. II s'agit de prôner un jeu de l'intériorité, naturaliste, " vrai », s'appuyant sur la mémoire affective et le subconscient. L'anglais Edward Gordon Craig (18721966) juge que l'humain est trop soumis au flux d'émotions changeantes. Comme les symbolistes, il pense que la solution réside dans la marionnette. Cette tendance à nier la sensibilité de l'acteur et à lui imposer un jeu de "surmarionnette " est finalement celle qui dominera toute la première moitié du XXe siècle.

7 C. Régy, "Commentaire dramaturgique ", [dans :] M. Maeterlinck, La Mort de Tintagiles. Théâtre. Commentaire dramaturgique de C. Régy, Arles, Actes Sud, 1997, p. 100.

$8 \mathrm{M}$. Maeterlinck, La Mort de Tintagiles, mise en scène C. Régy, scénographie D. Jeanneteau, avec Y. Boudaud, V. Dreville, V. Anton, Théâtre Gérard-Philippe, Saint Denis, 1997.

${ }^{9}$ M. Maeterlinck, La Mort de Tintagiles, mise en scène V. Meyerhold, Tiflis (Russie) 1907.
} 
options : «On peut présenter la pièce sous deux aspects tout à fait différents. Ou bien utiliser les paysages de Böcklin et les attitudes plastiques de Botticelli ; ou bien adopter le primitivisme des marionnettes ${ }^{10}$. Meyerhold sera déçu d'avoir choisi, trop tributaire des goûts de son époque, la première option, qui ne convient pas à une pièce si étrangère aux lois du théâtre de son temps. Mais c'est à partir de là que s'élabore sa divergence avec Stanislavski : alors que le premier forme des comédiens capables de tout jouer, le second préconise la spécialisation de l'acteur. Maeterlinck et Meyerhold ont une conception différente de la scène : le dramaturge belge joue le théâtre " contre " la scène, tandis que le metteur en scène russe exploite toutes les techniques de la scène. Néanmoins, selon Meyerhold, le théâtre de Maeterlinck contient une sagesse dans son mystère, et contient selon lui un "irrésistible appel à la vie ${ }^{11}$. Dans le contexte du totalitarisme soviétique, il affirme, à propos de la pièce : " Si l'on s'indigne, ce n'est pas contre la Mort, mais contre ce qui est porteur de mort ${ }^{12}$. II ne s'agit plus d'imiter la nature, mais de modifier le monde réel : l'artiste doit être capable " d'employer ses organes comme des instruments aptes à modifier selon sa fantaisie le monde réel ${ }^{13}{ }^{13}$, écrivait Novalis. En marge des idées de la Renaissance et des Lumières, Maeterlinck cherche à s'enraciner dans le romantisme allemand pour retrouver la relation entre la vie et I'homme intérieur : "Tout ce que nous appelons vie, univers, Dieu, n'est que notre façon de voir ce qui représente

\footnotetext{
10 V. Meyerhold, cité d'après G. Abensour, Vsévolod Meyerhold ou l'invention de la mise en scène, Paris, Fayard, 1998, p. 108.

11 V. Meyerhold, Le Théâtre théâtral, N. Gourfinkel (trad.), Paris, Gallimard, 1963, p. 38.

12 V. Meyerhold, cité d'après G. Abensour, Vsévolod Meyerhold ou l'invention de la mise en scène, op. cit., p. 107.

${ }^{13}$ G.-P. Novalis, Fragments, cité d'après P. Gorceix, [dans :] M. Maeterlinck, CEuvres I, op. cit., p. 259.
} 
une réalité que nous n'apercevrons pas tant que nous resterons ce que nous sommes $»^{14}$.

Il s'agit pour lui d'élaborer un nouveau vocabulaire, et ce vocabulaire radical, c'est Ruysbroeck qui le lui fournit. Ses prédécesseurs avaient bien senti l'importance que pourrait avoir l'influence de la langue des mystiques, et Meyerhold avait écrit que "le frémissement mystique est plus fort que le tempérament du vieux théâtre ${ }^{15}$. Mais Maeterlinck transpose dans son théâtre le langage de l'approximation et de l'indétermination caractéristiques du lexique mystique ${ }^{16}$, plaçant ses figures dans des situations qui dépassent la psychologie humaine.

Neuf ans avant l'écriture de La Mort de Tintagiles, la découverte de Ruysbroeck fut pour lui un choc. Alors qu'il se destinait à être avocat, entre 1885 et 1888, il se met à traduire L'Ornement des noces spirituelles et d'autres textes du mystique médiéval flamand. L'ouverture vers la mystique et l'ésotérisme se poursuit dans des rencontres décisives : celle de Villiers de I'Isle-Adam en 1886, et celle de Georgette Leblanc en 1895, cantatrice disciple de Gurdjieff avec laquelle il vivra pendant une vingtaine d'années (1895-1918). La Mort de Tintagiles date de 1894. Quant à Ruysbroeck, il devient le principe de sa Weltanschauung comme de sa poétique :

J'ai découvert (à peu près) un Ermite ou un Illuminé Flamand du XIIe siècle. Ruysbroeck l'Admirable, dont Ernest Hello a traduit en français presque scandaleusement d'ailleurs - certains fragments d'après une vieille et inexacte traduction latine du texte flamand ${ }^{17}$. [...] jamais je n'ai

\footnotetext{
${ }_{14}$ M. Maeterlinck, "Devant Dieu », [dans:] CEuvres I, op. cit., p. 237.

${ }^{15} \mathrm{~V}$. Meyerhold, Écrits sur le théâtre, cité d’après C. Régy, " Commentaire dramaturgique ", op. cit., p. 107.

16 Nous vous renvoyons à deux ouvrages fondamentaux sur l'étude du vocabulaire mystique: M. de Certeau, La Fable mystique I. XVle-XVIle siècles, Paris, Gallimard, 1982 et Pour un vocabulaire mystique au XVIIe siècle, séminaire du Professeur C. Ossola, textes réunis par F. Trémolières, Turin, Nino Argento, 2004.

17 J. van Ruysbroek, Rusbrock l'Admirable. CEuvres choisies, E. Hello (trad.), Paris, Perrin, 1921.
} 
éprouvé une joie ni un étonnement pareils, c'est l'homme de gé n ie a b s ol u et dont l'œuvre est immense matériellement - autrement surtout - cela est tout le temps au-dessus de tout, et cela va jusqu'où l'on n'a jamais été. ${ }^{18}$

Pour lui, " les écrits des mystiques sont les plus purs diamants du prodigieux trésor de l'humanité $»^{19}$. Ruysbroeck incarne "l'ignorance merveilleuse ${ }^{20}$ d'un esprit universel qui, au-delà des époques, des langues et des cultures, permet de rêver un syncrétisme de l'Orient et de l'Occident. Contenant toutes les traditions, il apporte aussi à Maeterlinck ses racines, la langue flamande : "Depuis que je l'ai vu, notre art ne me semble plus suspendu dans le vide. II nous a donné des racines ${ }^{21}$. Loin des traductions latines de Surius, Ruysbroeck a " à son usage un des modes du verbe presque originel, où les mots sont réellement des lampes derrière les idées, tandis que chez nous les idées doivent éclairer les mots $»^{22}$.

\section{Briser les catégories enfermantes de la pensée}

La Mort de Tintagiles, comme L'Oiseau bleu, traite d'une aventure dangereuse, celle d'une perte de soi qui rappelle l'expérience fondatrice de l'être au monde décrit par les grands mystiques de référence, et que Ruysbroeck qualifie $d^{\prime}$ " égarement sans retour ${ }^{23}$, proche du rapt, de l'enlèvement violent ${ }^{24}$, de la dévoration, pour qualifier un anéantissement de soi sans lequel aucun éveil, aucune nouvelle naissance n'est possible. Si Maeterlinck était

${ }_{18}$ M. Maeterlinck, "Avant-propos de Paul Gorceix », [dans :] CEuvres I, op. cit., p. 24.

19 Ibidem, p. 25.

${ }^{20}$ M. Maeterlinck, "Mystique, ésotérisme et écriture ", [dans :] CEuvres I, op. cit., p. 254.

21 Ibidem.

22 Ibidem.

23 J. van Ruysbroek, Rusbrock l'Admirable, op. cit., p. 110 : "Nous nous surpasserons nous-mêmes en hauteur, en profondeur et en largeur et ce sera quelque chose comme un égarement sans retour ".

${ }^{24}$ Ibidem, p. 201 : " Les hommes de la lumière sont librement ravis, plus haut que la raison, dans les domaines de la vision nue $"$. 
fasciné par ces mots du mystique flamand, c'est qu'ils donnent corps à sa dramaturgie : "Je suis englouti dans sa bouche / Je n'ai rien à faire au-dehors /... Oh ! quelle horreur vers le dehors! ${ }^{25}$. La pièce évoque en effet le pointcatastrophe de l'identité comme une aventure terrifiante, qui se caractérise par le choc angoissant des contraires. Dans La Mort de Tintagiles, on ne sait de quel côté sont le dedans et le dehors, ni le mal et le bien, tant sont floues les parois entre la terreur et le calme, entre l'ombre et la lumière, entre le savoir et l'ignorance. Même la vie et la mort n'apparaissent que comme des notions en mutation, comme l'a écrit ailleurs l'auteur: "Chaque homme n'est que le total provisoire d'un passé et d'un avenir qui se perd dans l'infini qui le précède et dans l'infini qui le suit ${ }^{26}$. Rien n'est résolu, et il n'est pas impossible qu'à l'instar des théories manichéennes, le bien et le mal ne soient pas séparés à l'intérieur de la divinité. De quel côté des portes de fer se trouve le mal ? Du côté de la Reine impitoyable et qui tue ? Faut-il voir les servantes comme des messagères du mal ? Faut-il lire la pièce comme une parabole de la lutte des bons contre les mauvais anges ? "Nous allons te défendre et le mal ne pourra pas venir ${ }^{27}$, dit Ygraine, dévorée par une obscure culpabilité, à son petit frère.

La Reine est présentée comme une créature à la fois maléfique et bénéfique, qui est l'ancêtre des personnages et séjourne dans une tour énorme que le temps n'attaque pas. Ygraine précise qu'elle dévore tout, mais épargne ceux qui s'agenouillent, et qu' « on ne sait pas sur quoi repose sa puissance ${ }^{28}$.

Elle est la mère de notre mère et elle veut régner seule... Elle est soupçonneuse et jalouse et on dit qu'elle est folle [...]. Ses ordres s'exécutent sans qu'on sache comment [...]. Elle a une puissance qu'on ne comprend pas et nous vivons ici avec un grand poids sans merci sur notre âme. ${ }^{29}$

\footnotetext{
25 Ibidem, p. 267.

${ }^{26}$ Ibidem, p. 222.

${ }_{27}$ M. Maeterlinck, La Mort de Tintagiles, op. cit., p. 22.

28 Ibidem, p. 18.

29 Ibidem, p. 13.
} 
Le dramaturge brise la logique habituelle en prêtant à ses figures des réactions surprenantes. À l'acte III, au moment où les deux sœurs tiennent l'enfant Tintagiles sur leurs genoux, tandis qu'Anglovale s'agrippe à son glaive, les portes s'ouvrent, cassant l'épée, et Tintagiles, contre toute attente, pousse un cri de délivrance. À l'acte IV, lorsque les trois servantes de la Reine viennent enlever l'enfant, elles le font lentement et sans brutalité, coupant délicatement les cheveux de ses sœurs endormies qui le retiennent dans son sommeil. On ne sait si cette scène signifie l'entrée dans une nouvelle vie ou le début d'une agonie, on ne sait si l'on assiste à un rite de mort (le rapt) ou de naissance (la coupure du cordon ombilical).

Parlant de l'âme qu'il aimerait placer au centre de son théâtre, Maeterlinck écrivait en 1896 : "On dirait qu'elle se trouve acculée à un mur invisible, et l'on ne sait si c'est l'agonie ou une vie nouvelle qui l'agite ${ }^{30}$. L'enfant Tintagiles, comme les autres personnages, illustre un moment de la vie de l'âme.

La ligne de démarcation entre le dedans et le dehors étant subvertie, les portes fonctionnent comme des allégories de la vie intérieure. Chez le lecteur assidu de Grimm, chaque porte renvoie à la chambre de Barbe-bleue ${ }^{31}$. Dans La Mort de Tintagiles, seul l'enfant peut franchir la grande porte de fer, à condition qu'il soit dans un état intermédiaire entre la veille et le sommeil. Dans L'Oiseau bleu également s'ouvriront devant les enfants une multitude de portes, mais il ne faut pas ouvrir la porte du milieu, celle derrière laquelle se cache l'oiseau bleu, le grand secret des choses et du bonheur. La porte, c'est aussi le rideau du théâtre, mais c'est surtout une transposition scénique de l'espace intérieur des mystiques. "Nous appelons âme ce

30 M. Maeterlinck, "Le Réveil de l'âme ", [dans:] CEuvres I, op. cit., p. 307. ${ }^{31}$ M. Maeterlinck s'en explique dans "Sur le théâtre ", [dans :] CEuvres I, op. cit., p. 442-445. 
que notre pensée n'atteint pas ${ }^{32}$, résume Maeterlinck. Les puissances de l'âme, toujours négatives car invisibles, sont mises en espace : le symbole de la porte de l'inconnaissable hante tout son théâtre. Dans La Grande Porte, il écrit : "c'est le désordre seul que je suis capable d'imiter "33. Parlant du désespoir d'Ygraine devant la porte, il précise que « nous sommes tous en attente devant cette porte qui ne sépare pas seulement la vie de la mort, mais le passé de l'avenir, le connu de l'inconnu et l'homme de son Dieu. J'y ai meurtri mes mots et mes pensées, comme Ygraine $s^{\prime} y$ meurtrissait les mains, et la porte ne s'est pas ouverte ${ }^{34}$. Dans La Mort de Tintagiles, il y a trois portes, une grande et deux petites. Qui ne s'ouvrent jamais. Elle sont fermées et scellées ${ }^{35}$. À l'acte IV seulement, elles dévoilent à la dérobée un corridor, un espace labyrinthique. L'écriture de Maeterlinck, comme l'espace scénique créé par Claude Régy, dramatisent à l'extrême la figure du seuil, qui est également une donnée constitutive du personnage principal. Ce seuil est un espace qui brise les associations convenues, exacerbe la tension des contraires, et matérialise les puissances invisibles : "On dit ceci, on dit cela, mais c'est l'âme qui suit un tout autre chemin " $^{36}$, écrit Maeterlinck.

Le thème du seuil est un leitmotiv des récits d'édification spirituelle, dont Le Château intérieur de Thérèse d'Avila représente un modèle du genre. La spatialisation de la vie intérieure est un lieu commun des écrits des mystiques. Dans Le Château intérieur, chaque étage réserve un seuil, et le septième mène à l'espace paradoxal d'une prison lumineuse, épreuve finale du pur amour, où l'exercitant se fond dans la foi divine en abandonnant les prérogatives

32 M. Maeterlinck, "Pensées, fragments, aphorismes », [dans :] CEuvres I, op. cit., p. 226.

33 lbidem, p. 240.

34 Ibidem, p. 239.

${ }^{35}$ M. Maeterlinck, La Mort de Tintagiles, op. cit., p. 20.

36 Ibidem, p. 35. 
de l'égo. Avec ses portes closes ou non closes, ses labyrinthes, ses impasses, le château de la Reine, à l'image du Château intérieur de Thérèse d'Avila, constitue un espace vivant, organique : " on dirait que les voûtes remuent "37, dit Ygraine. Ce n'est pas l'intérieur gothique flamboyant d'Äxe/38, ce drame de Villiers de l'Isle-Adam tant admiré de Maeterlinck. Ici tout est de plain-pied, pas d'étage, seule la tour surplombe. Cette notion du seuil ne peut s'approcher que dans la figure de l'oxymore : " Y a-t-il un moment où nous pouvons entendre la reine aux lèvres closes ? " ${ }^{39}$. Ce qui est cherché ne peut être attrapé. Maeterlinck situe l'action scénique dans un espace hors de la pensée dualiste. Selon Artaud, il réduit à néant le rationalisme matérialiste :

Il a su nous rendre sensibles [...] les lois occultes des phénomènes de la vie. Avec lui on a vraiment la sensation de descendre au fond du problème. La personne de Dieu est inconnaissable, dit la sagesse du Talmud, mais ses voies s'expriment par nombres et par chiffres. Ce sont ces nombres dont la nature est insensible maintenant au commun que Maeterlinck a fixés en phrases lapidaires. ${ }^{40}$

Mais le théâtre de Maeterlinck, s'il s'appuie sur la contradiction assumée, est dans un premier temps marqué par la terreur, ou du moins la violence indissociable de l'élan vital. À bien des égards, son théâtre annonce ce qu'Artaud nommera "le théâtre de la cruauté ", et qu'il identifiait " avec cette sorte de cruauté vitale qui est à la base de la réalité ${ }^{41}$. Le rapt de l'enfant dans La Mort de Tintagiles, la hantise de la dévoration, le sectionnement du cordon ombilical matérialisé par les cheveux des sœurs de l'enfant relèvent de cette cruauté, qui renvoie à la bru-

\footnotetext{
37 Ibidem, p. 34.

${ }^{38}$ A. de Villiers de I'Isle-Adam, Äxel, Nancy, Absalon, 2008.

${ }^{39} \mathrm{M}$. Maeterlinck, "Mystique, ésotérisme et écriture ", [dans :] CEuvres I, op. cit., p. 314.

${ }^{40} \mathrm{~A}$. Artaud, cité d'après C. Régy, "Commentaire dramaturgique ", op. cit., p. 48.

${ }^{41}$ A. Artaud, Le Théâtre et son double, Paris, Gallimard, 1984, p. 176-177.
} 
talité de toute naissance, réelle ou symbolique. Dans $L a$ Mort de Tintagiles s'affirme un théâtre qui fait de " la terreur [...] l'atmosphère-même du poème ${ }^{42}$. De même que la peur et le désir sont les deux faces de l'action, l'action dramatique est remplacée par la contemplation, qui fait du concept d' " acteur " un contresens. Que veut dire en effet " agir " dans un théâtre où la passivité active est toute-puissante.

Dans son célèbre texte Le tragique quotidien (1886), Maeterlinck se livre à une refonte de l'écriture dramatique qui sera celle du théâtre statique, et qui supprime l'action pour rendre compte des mouvements peu perceptibles de la conscience.

Est-il donc hasardeux d'affirmer que le véritable tragique de la vie, le tragique normal, profond et général, ne commence qu'au moment où ce que nous appelons les aventures, les douleurs et les dangers sont passés ? Le bonheur n'aurait-il pas le bras plus long que le malheur et certaines de ses forces ne s'approcheraient-elles pas davantage de l'âme humaine ? [...] N'est-ce pas la tranquillité qui est terrible lorsqu'on y réfléchit et que les astres la surveillent ? N'est-ce pas quand on nous dit à la fin des histoires " ils furent heureux " que la grande inquiétude devrait commencer ? [...] N'est-ce pas quand un homme se croit à l'abri de la mort extérieure que l'étrange et silencieuse tragédie de l'être et de son immensité ouvre vraiment les portes de son théâtre ?43

Selon lui, le vieillard immobile à sa fenêtre vit d'une vie plus profonde que celle du criminel :

Subissant la présence de son âme et de sa destinée, inclinant un peu la tête, sans se douter que toutes les puissances de ce monde interviennent et veillent dans la chambre comme des servantes attentives, ignorant que le soleil lui-même soutient au-dessus de l'abîme la petite table sur lequel il s'accoude, et qu'il n'y a pas un astre du ciel ni une force de l'âme qui ne soient indifférents au mouvement d'une paupière qui retombe ou d'une pensée qui s'élève. ${ }^{44}$

Or, l'effroi de la contemplation de l'essentiel est lié au regard que les mystiques portent sur le réel, comme Mae-

\footnotetext{
42 M. Maeterlinck, "Menus propos », [dans:] CEuvres I, op. cit., p. 463.

${ }^{43} \mathrm{M}$. Maeterlinck, "Le tragique quotidien ", [dans:] CEuvres I, op. cit., p. 487-488.

44 Ibidem, p. 490.
} 
terlinck l'écrit dans la préface à sa traduction de Ruysbroeck, présenté comme celui « qui habitait à l'horizon de l'homme ${ }^{45}$, qui est allé jusqu'à l' « l'instant où l'amour devenait strictement effrayant ${ }^{46}$, et nous enseigne "le saint mal des joies au-delà de nos cœurs $»^{47}$ car, en fixant " l'inouï qu'il révèle avec calme ${ }^{48}$, il a osé " entrouvrir les symboles de certaines portions de ces deux effrayants et inexplorables symboles de nul ne sait quoi : l'espace et le temps ${ }^{49}$.

Si la pièce La Mort de Tintagiles peut paraître relever d'un théâtre de la cruauté avant l'heure, elle traduit surtout la recherche d'un nouveau vocabulaire, à la fois textuel et scénique, pour faire du plateau du théâtre un espace de manifestation des réalités invisibles ou impensées, propres à la vie intérieure, aux deux extrémités de la vie humaine, qu'elles concernent l'enfance ou la vieillesse. Maeterlinck est de ceux qui considèrent que le théâtre est un espace-temps destiné à faire vivre aux spectateurs une expérience qu'ils ne peuvent vivre ailleurs, et qui les relie à leur être intime, insaisissable, celui-là même qui fonde notre relation au réel, aux autres. Cette façon de pratiquer le théâtre se poursuit aujourd'hui à travers les récents propos du grand metteur en scène italien Roméo Castellucci, lors d'un entretien sur France Culture en 2019 : " l'acte politique, c'est d'inventer des lieux qui n'existent pas. D'abord sortir, s'éloigner. Le théâtre, c'est un grand combat contre la réalité, pour créer du réel $»^{50}$.

Date de réception de l'article : 17.11.2019. Date d'acceptation de l'article : 07.01.2020.

${ }^{45} \mathrm{M}$. Maeterlinck, préface à J. Van Ruysbroek, L'Ornement des noces spirituelles de Rusbroeck l'Admirable, M. Maeterlinck (trad.), Bruxelles, Eperonniers, 1990, p. 283.

$46 \mathrm{lbidem}$, p. 283.

47 Ibidem, p. 284.

$48 \mathrm{lbidem}$, p. 274.

49 Ibidem, p. 283.

50 R. Castellucci, entretien avec O. Gesbert dans " La Grande table », France culture, 20 novembre 2019, durée 26 mn. https://www.franceculture.fr/ emissions/la-grande-table-culture/romeo-castellucci-maitre-de-ceremonie. 


\section{bibliographie}

Abensour G., Vsévolod Meyerhold ou l'invention de la mise en scène, Paris, Fayard, 1998.

Artaud A., Le Théâtre et son double, Paris, Gallimard, 1984.

Brook P., L'Espace vide. Écrits sur le théâtre, Paris, Seuil, 1968.

Castellucci R., entretien avec O. Gesbert dans "La Grande table ", France culture, 20 novembre 2019, https://www.franceculture.fr/emissions/lagrande-table-culture/romeo-castellucci-maitre-de-ceremonie.

Certeau M. de, La Fable mystique I. XVle-XVIIe siècles, Paris, Gallimard, 1982.

Maeterlinck M., L'Oiseau bleu, [dans :] Idem, CEuvres III. Théâtre 2, P. Gorceix (éd. critique), Bruxelles, Complexe, 1999.

Maeterlinck M., Les Débris de la guerre, Paris, Fasquelle, 1916.

Maeterlinck M., CEuvres I. Le Réveil de l'âme. Poésie et essais, P. Gorceix (éd. critique), Bruxelles, Complexe, 1999.

Maeterlinck M., La Mort de Tintagiles. Théâtre. Commentaire dramaturgique de C. Régy, Arles, Actes Sud, 1997.

Maeterlinck M., La Mort de Tintagiles, mise en scène C. Régy, scénographie D. Jeanneteau, avec Y. Boudaud, V. Dreville, V. Anton, Théâtre Gérard-Philippe, Saint Denis, 1997.

Maeterlinck M., La Mort de Tintagiles, mise en scène V. Meyerhold, Tiflis, Russie, 1907.

Meyerhold V., Le Théâtre théâtral, N. Gourfinkel (trad.), Paris, Gallimard, 1963.

Parisse L., Les Voies négatives de l'écriture dans le théâtre moderne et contemporain, Paris, Classiques Garnier, 2019.

Parisse L. (dir.), Le Discours mystique dans la littérature et les arts de la fin du XIXe siècle à nos jours, Paris, Classiques Garnier, 2012.

Ruysbroek J. Van, Rusbrock I'Admirable. CEuvres choisies, E. Hello (trad.), Paris, Perrin, 1921.

Ruysbroek J. Van, L'Ornement des noces spirituelles de Rusbroeck l'Admirable, M. Maeterlinck (trad.), Bruxelles, Eperonniers, 1990.

Trémolières F. (dir.), Pour un vocabulaire mystique au XVIle siècle, séminaire du Professeur C. Ossola, Turin, Nino Argento, 2004.

Villiers de I'Isle-Adam A. de, Äxel, Nancy, Absalon, 2008.

\section{abstract}

\section{Maeterlinck's Innertheater in Tintagile's death}

Tintagile's death (1894) is an etape in the Maeterlinck's work. This drama bears the strong imprint of a decisive évent in the life of the author : the discovery of the writings of the médieval Flemish mystic Ruysbroeck, of wich he became the translator from 1885 to 1888 . The langage of mysticism allows a renowal of writing and dramatic aesthetics. 


\section{keywords}

Maeterlinck, Ruysbroeck, théâtre intérieur, langue de la mystique

\section{mots-clés}

Maeterlinck, Ruysbroeck, Innertheater, language of mysticism

\section{lydie parisse}

Lydie Parisse est maître de conférences de littérature française habilitée à diriger les recherches en arts du spectacle, à l'Université de Toulouse 2 . Écrivaine et metteuse en scène, ses pièces sont publiées, dont une en Pologne (trad. T. Swoboda, 2018). Aux Classiques Garnier : Mystique et littérature. L'Autre de Léon Bloy (2019 [2006]) ; Le Discours mystique dans la littérature et les arts de la fin du XIXe siècle à nos jours (collectif, 2012); Lagarce. Un théâtre entre présence et absence (2014) ; La Parole trouée. Beckett, Tardieu, Novarina (2019 [2008]) ; Les Voies négatives de l'écriture dans le théâtre moderne et contemporain (2019). https://plh.univ-tlse2.fr ORCID : https://orcid.org/0000-0002-3081-8175 